\title{
Comparación de rendimiento de antenas microstrip con diferentes frecuencias y polarización
}

\section{Performance comparison of microstrip antennas with different frequencies and polarization}

Galo Hurtado Crespo. ${ }^{1}$, Juan Pérez Pérez. ${ }^{2} \&$ Zoila Contreras Paguay. ${ }^{3}$

\begin{abstract}
.
DOI: https://doi.org/10.33262/cienciadigital.v4i3.1304

The research work is based on the analysis of the operation of microstrip antennas for which three different antennas were designed, working in bands ranging from $800 \mathrm{MHz}$ - to $5.5 \mathrm{GHz}$, checking that the functionality is efficient and that they do not interfere with other frequency bands, There are many advantages that can be taken into consideration when implementing antennas with microstrip technology, such as cost, easy manufacturing, works at high frequencies and sufficient amount of information in the different repositories, to be able to perform the tests, first you have to design the model of the antenna in the software Ansoft Designer, then has to perform the analysis of frequency resonance and radiation pattern, determining the frequency at which it works, as well as the bandwidth and gain of each of them, once verified the proper functioning of the antennas proceed to the manufacture of the antenna, you have to select the material, the thickness of the material, as well as the connectors and the welding points that are made, all these factors influence the optimal operation of the antennas, finally to be able to make the field tests it is necessary to work with a radio spectrum analyzer, the same one generates different signals with different gains and impedances, that will allow to determine in which band of frequencies it is working.
\end{abstract}

Keywords: Antennas, microstrip, polarization, frequencies, radio spectrum.

\footnotetext{
${ }^{1}$ Docente investigador, Cuenca, Ecuador, ORCID id: http://orcid.org/0000-0002-7190-140X Email: gphurtado@sudamericano.edu.ec

2 Director de Carrera, Cuenca, Ecuador. ORCID id: https://orcid.org/0000-0002-8681-9855, jperez@sudamericano.edu.ec

${ }^{3}$ Estudiante, Cuenca, Ecuador. ORCID id: https://orcid.org/0000-0002-8338-6871, Email: zmcontreras@sudamericano.edu.ec
} 


\section{Resumen.}

El trabajo de investigación se basa en el análisis del funcionamiento de las antenas microstrip para lo cual se realizó el diseño de tres antenas diferentes, que trabajen en bandas que van desde los $800 \mathrm{MHz}$ - hasta los 5,5 GHz comprobando que la funcionalidad sea eficiente y que no interfieran con otras bandas de frecuencias, existen muchas ventajas que se pueden tomar en consideración al momento de implementar antenas con la tecnología microstrip, como son el costo, la fácil fabricación, trabaja a grandes frecuencias y la suficiente cantidad de información en los diferentes repositorios, para poder realizar las pruebas, primero se tiene que diseñar el modelo de la antena en el software Ansoft Designer, posteriormente se tiene que realizar el análisis de resonancia de frecuencias y patrón de radiación, determinando la frecuencia en la que trabaja, así como el ancho de banda y la ganancia de cada una de ellas, una vez comprobado el correcto funcionamiento de las antenas se procede a la fabricación de la antena, se tiene que seleccionar el material, el grosor del material, así como los conectores y los puntos de suelda que se realicen, todos estos factores influyen en el óptimo funcionamiento de las antenas, finalmente para poder realizar las pruebas de campo es necesario trabajar con un analizador de espectros radioeléctrico, el mismo generar diferentes señales con ganancias e impedancias diferentes, que permitirán determinar en qué banda de frecuencias está trabajando.

Palabras clave: Antenas, microstrip, polarización, frecuencias, espectro radioeléctrico.

\section{Introducción.}

En la actualidad la información es un derecho al que todos los ciudadanos pueden, acceder por diferentes medios (Villanueva, 2006), por lo tanto, tienen que existir los medios de comunicación que permitan acceder a la información, con los avances tecnológicos han ido surgiendo nuevas maneras de comunicarse, para poder llegar a lugares remotos que en la antigüedad acceder a la información era imposible, ya sea por condiciones económicas, de distancias o de tecnología, con la finalidad de solventar estas necesidades surgen dispositivos que adaptan ondas guiadas, desde conductores o guías al espacio libre conocidas como antenas microstrip (Vielma, 2005). Esta tecnología funciona en la banda de 2,4 GHz en la mayoría de los países y trabaja en la banda de frecuencia de $5 \mathrm{GHz}$. La señal que se propaga en ambientes interiores afectados por diversas reflexiones y procesos complejos de dispersión (procesos asociados a la dispersión experimentada por la señal), de modo que la polarización de la señal a lo largo de la transmisión puede variar (Balanis, 2005). Para el caso de estudio se realizó el diseño, cálculo matemático, implementación y comparación de la ganancia y perdida de cada una de ellas, partiendo de las antenas microstrip Dipolo doble que se han convertido en la actualidad en un tema de gran importancia para los investigadores, especialmente sus aplicaciones en sistemas de comunicación por satélite, móviles e inalámbricos, debido a su pequeño tamaño, su peso ligero y su facilidad de producción. Estas antenas, en general, resuenan eficientemente en una sola frecuencia de resonancia que corresponde a su modo dominante y tienen 
típicamente un ancho de banda estrecho y baja ganancia (Luberto \& Fano, 2016). Se han llevado a cabo pruebas, para mejorar la funcionalidad de las antenas de patch circulares, con la inclusión de ranuras de geometrías concéntricas como diamante, triangulares, cuadradas, teniendo la particularidad de que al ser integradas como una ranura en la antena de parche se obtiene un mejor ancho de banda según se menciona en el artículo (Diseño antena microstrip de ranura y antena de parche con geometría fractal, n.d.), y lo más importante la capacidad de trabajar en dos o más frecuencia, la banda S y C (Cabedo \& Puente, n.d.), específicamente en comunicaciones satelitales e inalámbricas, por qué está diseñada para una frecuencia de $2,5 \mathrm{GHz}$ y $4,5 \mathrm{GHz}$. Y la tercera antena en el caso de estudio fue una antena trabando que trabaje en las frecuencias de 2,5 GHz, 3,6 GHz, y $5.5 \mathrm{GHz}$ la intención es comparar el rendimiento de las diferentes antenas (Borja Borau, 2001).

\section{Materiales y métodos.}

La investigación se realizó atreves de una metodología experimental con un análisis cuantitativo para poder evaluar los resultados, en la gráfica se pretende estudiar cómo el plano del suelo afecta al dipolo básico, el hecho de tener un plano de tierra afecta a la respuesta del dipolo (Bernal, 2008). Teniendo en cuenta cómo afecta tener un plano de tierra, se ha realizado un estudio para ver si las dimensiones de este tienen un efecto en la respuesta del dipolo. Para ello se hizo una comparación entre el plano de tierra utilizado con dimensiones 33x33mm.En la antena de diseño la antena circular de parche tiene un radio de $16,2 \mathrm{~mm}$; con dos frecuencias 2,5 y 4,5 GHz en la banda $\mathrm{S}$ y $\mathrm{C}$, con plano de tierra infinito y las dimensiones de la ranura en forma de diamante son $a=4 \mathrm{~mm}$ y $b=8 \mathrm{~mm}$, donde a es el valor medio de la anchura del diamante, como se muestra en las siguientes tablas (Firmansyah et al., 2016). Y la antena de tres bandas tiene una longitud de 48,51 $\mathrm{mm}$, con una separación de $20.76 \mathrm{~mm}$, cabe recalcar que todas las antenas se realizaron mediante una simulación en el software Ansoft Designer (Safari \& Shafai, 2009), para observar el comportamiento y comprobar cuál es el rendimiento de acuerdo a los tres diseños de antenas planeadas para el caso de estudio.

Figura 1. Diseño de antenas en Ansoft Designer

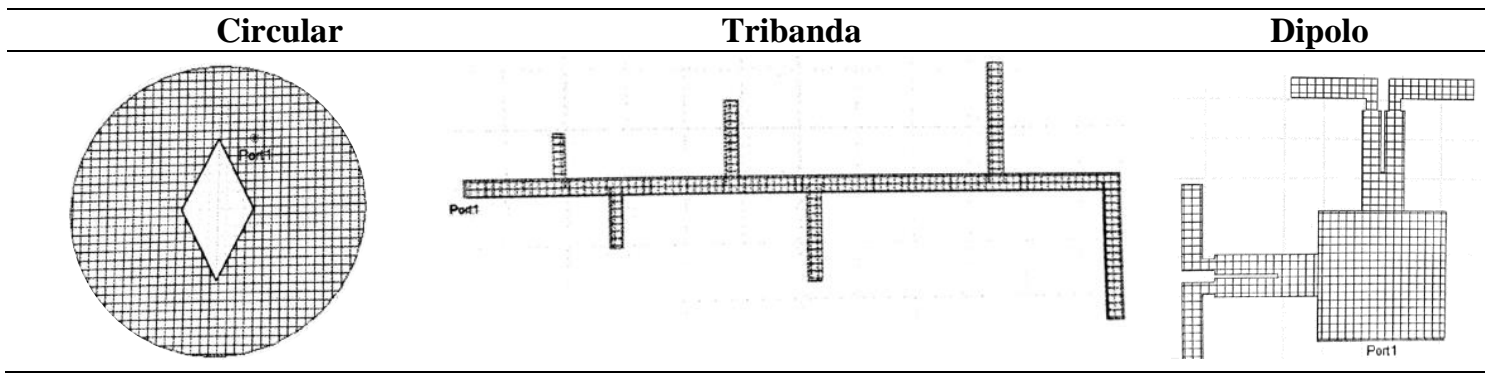

Fuente: (Hurtado-Crespo, 2020) 
Una vez diseñadas las antenas con las medidas y parámetros correspondientes se realizan el análisis de la frecuencia a la que trabajara y con la ayuda de los diagramas de radar, en 2D se analizara también la dirección de propagación del campo eléctrico, la ganancia, la polarización de la antena (dirección de la onda radiada).

Se realizó la simulación de las antenas con los diagramas de radiación obteniendo como resultado las siguientes graficas:

Figura 2. Antena Patch resonancia de frecuencias y patrón de radiación

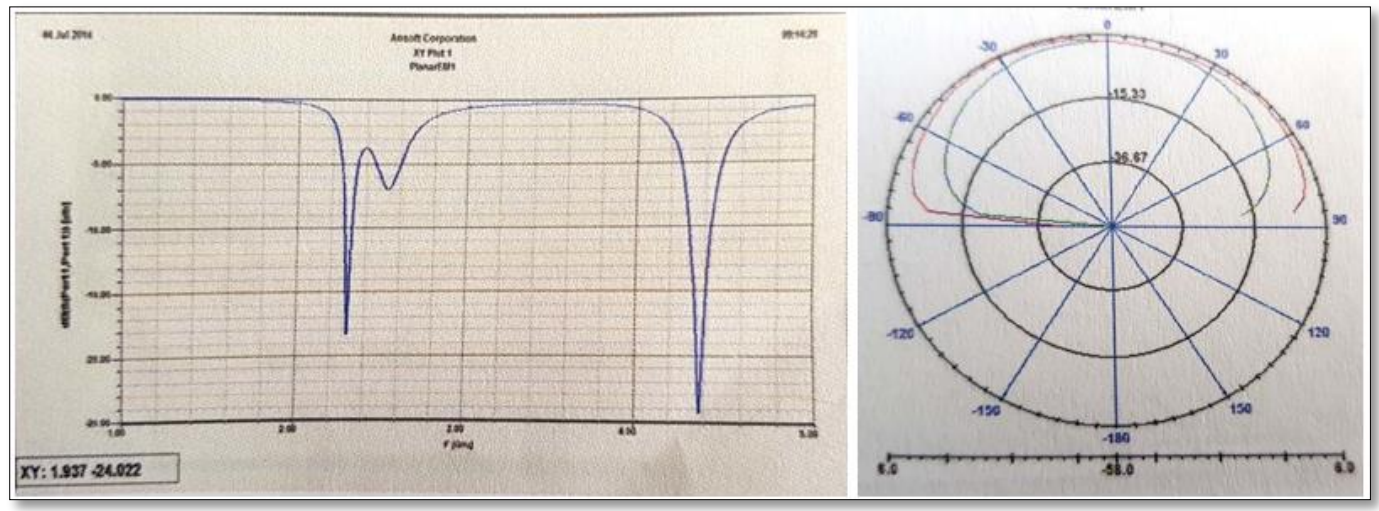

Fuente: (Hurtado-Crespo, 2020)

Como se muestra en la imagen se puede observar la impedancia y como trabaja en las diferentes frecuencias además se puede establecer un funcionamiento óptimo en el diseño de la antena, para de esta manera poder pasar al siguiente paso que sería la de imprimir la antena para posteriormente realizar ya pruebas de campo (Rao et al., 2003).

Figura 3. Antena Dipolo resonancia de frecuencias y patrón de radiación

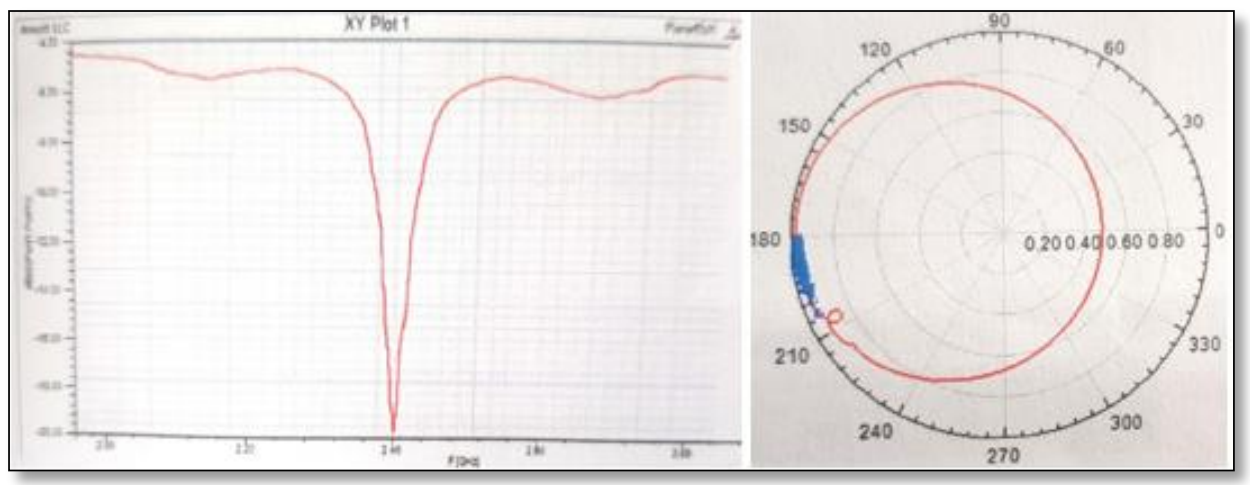

Fuente: (Hurtado-Crespo, 2020)

Esta simetría en las líneas de energía asegura que la respuesta para el dipolo vertical y el dipolo horizontal será muy similar. El propósito del diseño es también que el rendimiento 
de los dos dipolos sea similar a que no haya diferencias en el tiempo de recepción de la señal (Huidobro, 2013). Sin embargo, a pesar de tener una desviación en la frecuencia de adaptación, dos dipolos tienen una anchura de banda similar en la que la adaptación tiene un valor inferior a - $10 \mathrm{~dB}$.

Figura 4. Antena Tribanda (Yagui) resonancia de frecuencias y patrón de radiación, Frecuencia de $2.4 \mathrm{GHz}$

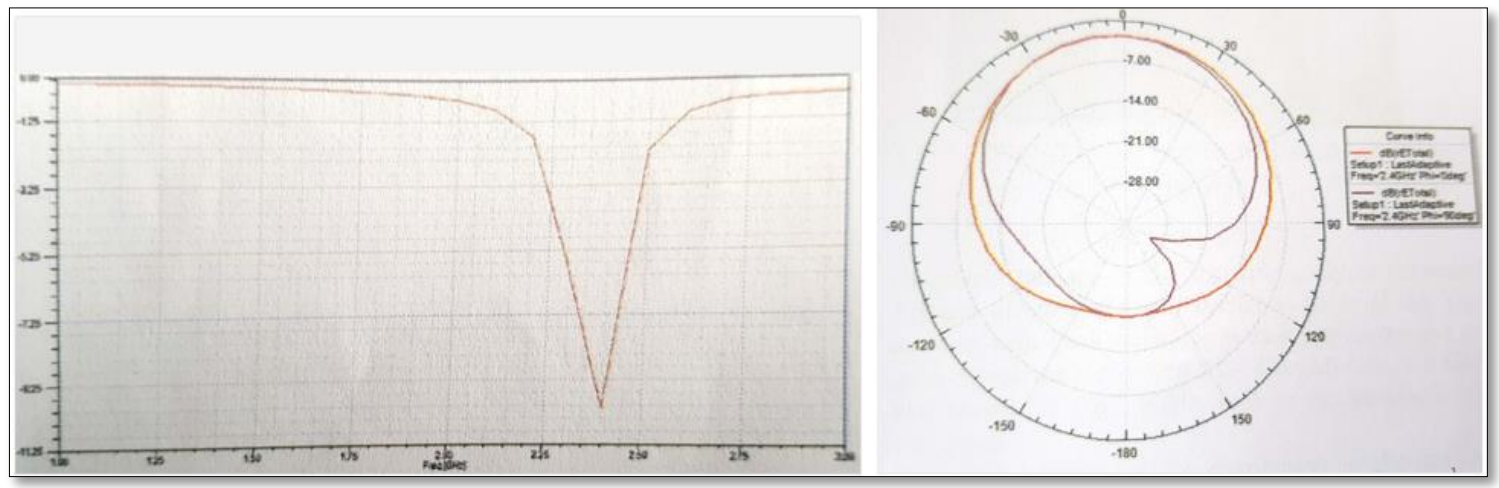

Fuente: (Hurtado-Crespo, 2020)

Figura 5. Antena Tribanda (Yagui) resonancia de frecuencias y patrón de radiación, Frecuencia de $3.6 \mathrm{GHz}$

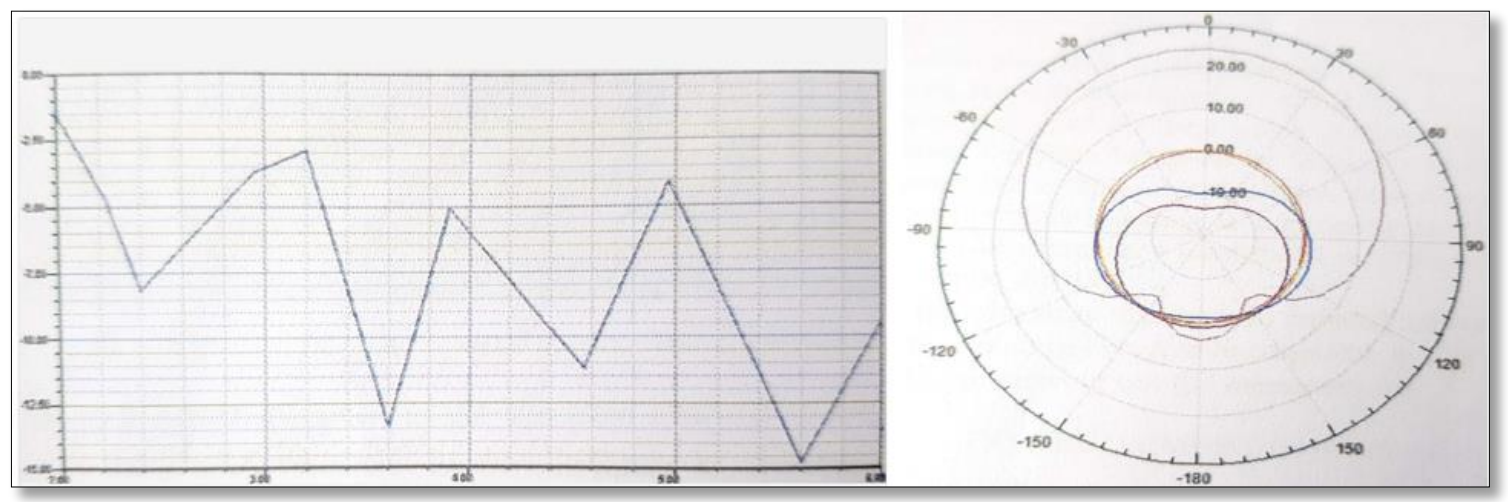

Fuente: (Hurtado-Crespo, 2020) 
Figura 6. Antena Tribanda (Yagui) resonancia de frecuencias y patrón de radiación, Frecuencia de $5.5 \mathrm{GHz}$

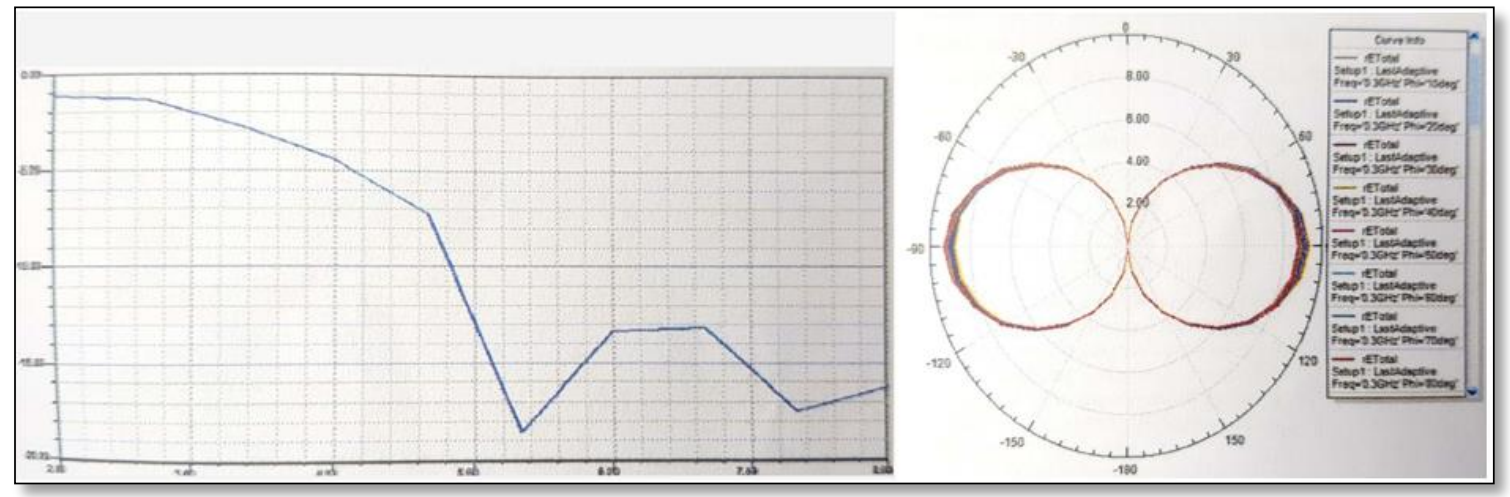

Fuente: (Hurtado-Crespo, 2020)

Se realizó el análisis en cada una de las frecuencias obteniendo como resultado, la representación de los lóbulos de radiación trabajando en un estado óptimo.

\section{Resultados.}

Una vez realizadas las respectivas simulaciones y análisis de resultados se procede a la fabricación de las antenas microstrip obteniendo los siguientes resultados:

Figura 7. Antena Patch Diseño y recepción de señal

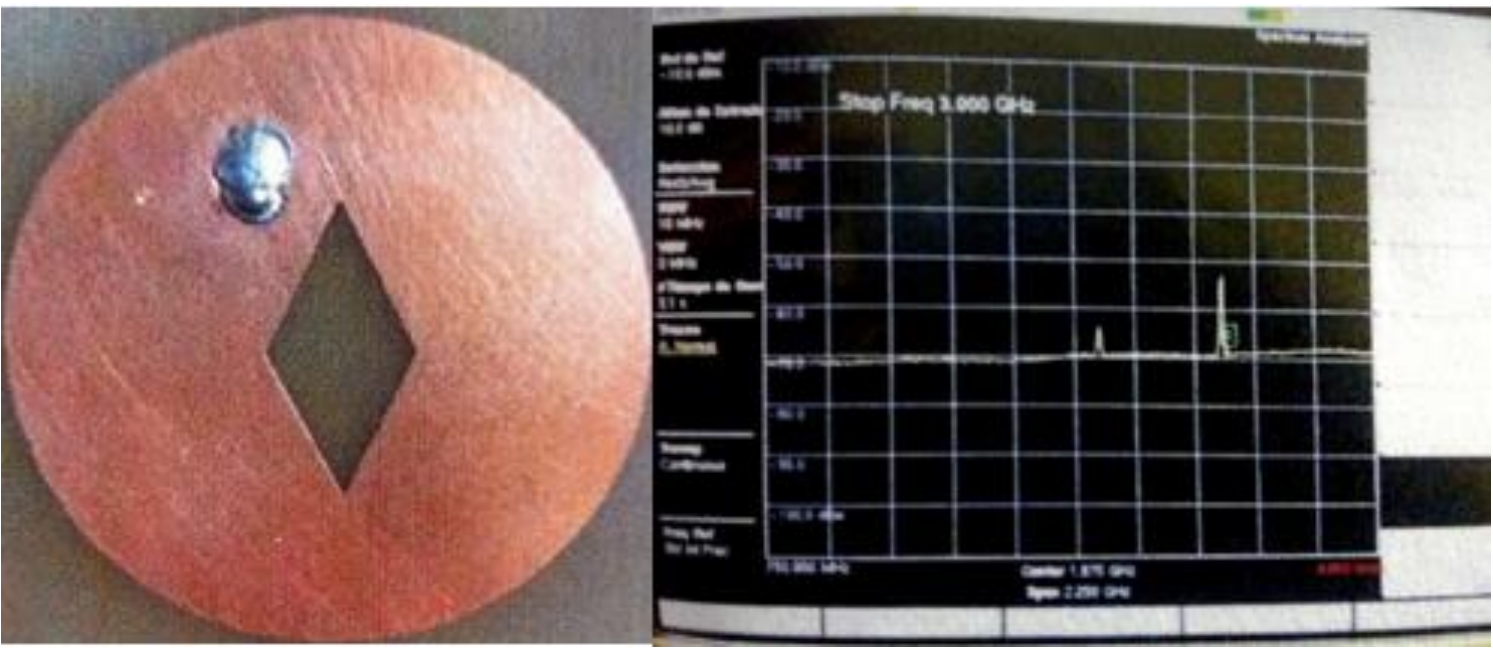

Fuente: (Hurtado-Crespo, 2020)

Las pruebas realizadas en el analizador demuestran que la antena opera para telefonía celular en un rango de $800 \mathrm{Mhz}-900 \mathrm{MHz}, 2.4 \mathrm{GHz}$ y $1.8 \mathrm{GHz}$, se observó que no hay recepción de la antena telefónica en las mismas frecuencias por lo tanto la antena se podría utilizar para transmitir diferente tipo de información en esas bandas libres sin generar 
interferencia con ninguna de las de telefonía celular ya que las bandas de frecuencias de operación son diferentes (Huidobro, 2013).

Figura 8. Antena Dipolo Diseño y recepción de señal

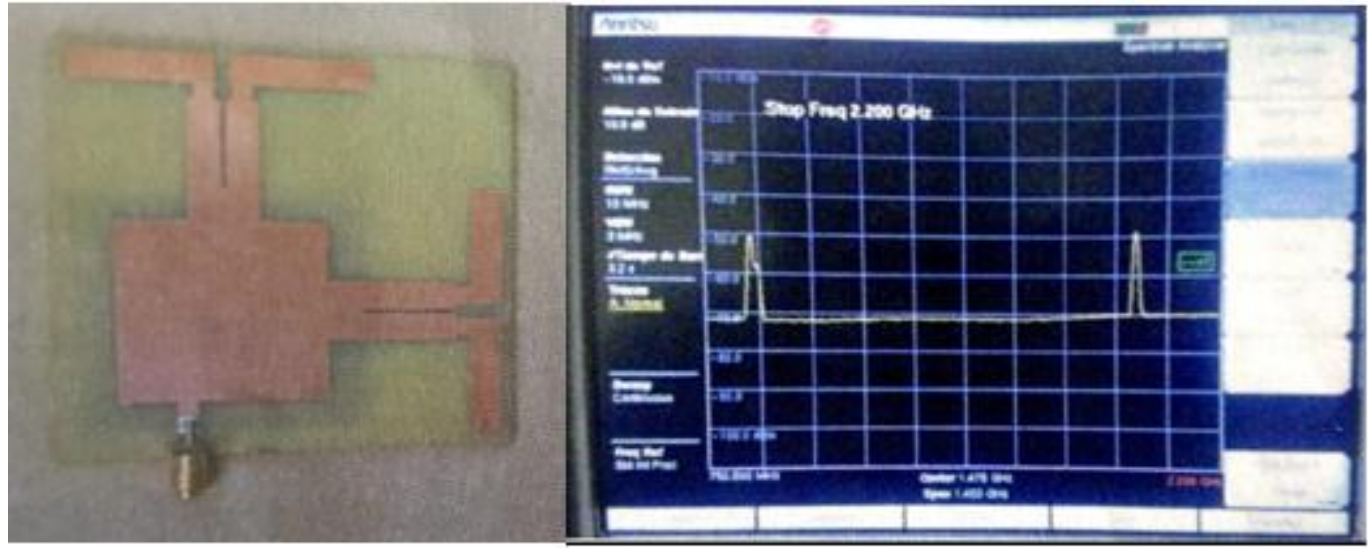

Fuente: (Hurtado-Crespo, 2020)

Se puede ver que hay un pico a $-25 \mathrm{~dB}$ que indica que detecta una señal que opera a la frecuencia especificada, es decir que trabaja a la misma frecuencia que una de telefonía celular generando interferencia, y desde un rango de $800 \mathrm{Mhz}$ - $1 \mathrm{GHz}$ se puede observar que no detecta ninguna señal y es debido a que la antena opera a una frecuencia de $2.4 \mathrm{Ghz}$.

Figura 9. Antena Patch Diseño y recepción de señal

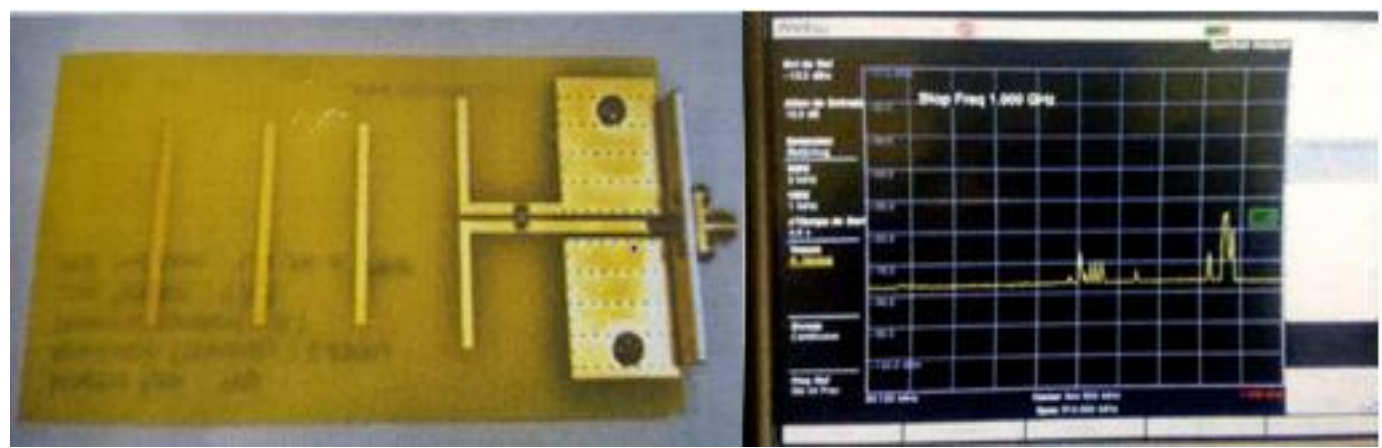

Fuente: (Hurtado-Crespo, 2020)

Se realizó el análisis en las frecuencias de 2,4 GHz, 3,6 GHz, y 5,5 GHz como se muestra en la gráfica el resultado del análisis en $2,4 \mathrm{GHz}$, que es la frecuencia que se realizaron la mayoría de pruebas y se determinó que no existe una interferencia de frecuencias y que al trabajar hasta la banda de $5 \mathrm{GHz}$ se puede aprovechar de una mejor manera ya que en la actualidad la mayoría de dispositivos móviles trabajan en ese rango de frecuencias optimizando la velocidad de transmisión delos datos. 


\section{Conclusiones:}

- Discusión, las antenas de tipo patch son dispositivos miniaturizados, que hoy en día son una herramienta extremadamente importante para utilizar en sistemas de comunicación, todo dependerá de un buen diseño y posteriormente de realizar las respectivas pruebas para proceder a la fabricación, una de las ventajas más importantes que presenta es que trabaja a grandes frecuencias y son de un costo muy accesible. Se realizó las pruebas en diferentes frecuencias con la finalidad de comprobar el rendimiento y funcionalidad de las tres antenas propuestas para el caso de estudio, se trabajó en las frecuencias que van desde los $800 \mathrm{MHz}$-hasta los 5,5 GHz comprobando si las antenas interfieren o no con otras bandas, todo esto se pudo determinar en el analizador de espectros radioeléctricos, de esta manera al momento de operar se evitan posibles sanciones que pueda emitir la ARCOTEL que es el ente regulador de frecuencias radioeléctricas del país.

\section{Referencias bibliográficas:}

Balanis, C. A. (2005). Antenna Theory: Analysis and Design, 3rd Edition. WileyInterscience. $\quad$ http://www.amazon.com/Antenna-Theory-Analysis-DesignEdition/dp/047166782X

Bernal, I. (2008). Revisión de Conceptos Básicos de Antenas y Propagación QuitoEcuador.

Borja Borau, M. C. (2001). Fractal microsttrip patch antennas with fractal perimeter and self-affine properties. 1.

Cabedo, A., \& Puente, C. (n.d.). DISEÑO DE ANTENAS MULTIBANDA PARA TELEFONÍA MÓVIL MEDIANTE LA MANIPULACIÓN DEL PLANO DE MASA.

Diseño antena microstrip de ranura y antena de parche con geometría fractal. (n.d.). Retrieved April 16, 2020, from http://academicae.unavarra.es/handle/2454/4806?show=full

Firmansyah, T., Purnomo, S., Fatonah, F., \& Fajar Nugroho, T. H. (2016). Antena Mikrostrip Rectangular Patch 1575,42 MHz dengan Polarisasi Circular untuk Receiver GPS. Jurnal Nasional Teknik Elektro Dan Teknologi Informasi (JNTETI), 4(4), 243-249. https://doi.org/10.22146/jnteti.v4i4.170

Huidobro, J. M. (2013). Antenas de telecomunicaciones.

Hurtado-Crespo, G. P. (2020). Estudio de factibilidad de políticas de seguridad iso/iec 27002:2013 para instituciones educativas adaptables a las pymes | International Journal of Recent Scientific Research. Recentscientific. https://doi.org/http://dx.doi.org/10.24327/ijrsr.2020.1012.4955

Luberto, M., \& Fano, W. G. (2016). Microstrip antenna design using EBG (Electromagnetic Band Gap) structures at 2.4GHz. 2015 16th Workshop on Information Processing and Control, RPIC 2015, 1-7. https://doi.org/10.1109/RPIC.2015.7497097 
Rao, B. R., Smolinski, M. A., Quach, C. C., \& Rosario, E. N. (2003). Triple-band GPS trap-loaded inverted L antenna array. Microwave and Optical Technology Letters, 38(1), 35-37. https://doi.org/10.1002/mop.10963

Safari, F., \& Shafai, L. (2009). A parametric study using ansoft HFSS and ansoft designer on the effect of aperture diameter on the transmission properties of its array. Proceedings - 2009 13th International Symposium on Antenna Technology and Applied Electromagnetics and the Canadian Radio Sciences Meeting, ANTEM/URSI 2009. https://doi.org/10.1109/ANTEMURSI.2009.4805108

Vielma, M. (2005). Introducción a las Antenas Contenido.

Villanueva, E. (2006). Derecho de la información. Editorial Miguel Ángel Porrúa. 


\section{PARA CITAR EL ARTÍCULO INDEXADO.}

Hurtado Crespo, G., Pérez Pérez, J., \& Contreras Paguay, Z. (2020). Comparación de rendimiento de antenas microstrip con diferentes frecuencias y polarización. Ciencia Digital, 4(3), 107-116. https://doi.org/10.33262/cienciadigital.v4i3.1304

\section{\Ciencia}

El artículo que se publica es de exclusiva responsabilidad de los autores y no necesariamente reflejan el pensamiento de la Revista Ciencia Digital.

El artículo queda en propiedad de la revista y, por tanto, su publicación parcial y/o total en otro medio tiene que ser autorizado por el director de la Revista Ciencia Digital.
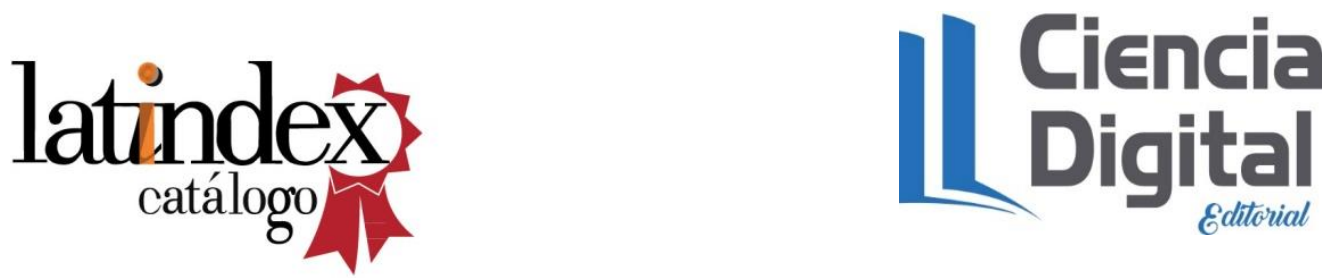